\title{
Performance Evaluation of Cross-Layer Routing for QoS Support in Mobile Ad Hoc Networks ${ }^{\star}$
}

\author{
María Canales, José Ramón Gállego, Ángela Hernández-Solana, \\ and Antonio Valdovinos \\ Institute of Engineering in Aragón, I3A, University of Zaragoza \\ C \María de Luna, 3, 50.018, Zaragoza (Spain) \\ mcanales@unizar.es, jrgalleg@unizar.es, anhersol@unizar.es, \\ toni@unizar.es
}

\begin{abstract}
Mobile ad hoc networks (MANETs) appear nowadays as one of the most promising architectures to flexibly provide multimedia services in multiple wireless scenarios. However, the dynamic nature of this environment complicates the supporting of the heavily demanded QoS. Since cooperation in MANETs is required to establish multihop communications, designing efficient QoS Routing algorithms mainly concentrates the technical efforts to guarantee QoS. This work presents a crosslayer architecture that performs a practical solution to solve the trade-off between the QoS provision and the efficient resource utilization thanks to different layers sharing network status information to cooperate in the network resource management. The cooperation between Routing and MAC levels allows to select End-to-End QoS paths according to the bandwidth availability measured in a realistic interference scenario, and appropriately react to mobility in a QoS context.
\end{abstract}

\section{Introduction}

Mobile ad hoc networks (MANETs) appear nowadays as one of the most promising architectures to flexibly provide multimedia services in multiple wireless scenarios. However, the dynamic nature of this environment makes it difficult to support the heavily demanded QoS. Cooperation in MANETs is required to establish multihop communications, relying on the nodes capability to act both as host and routers. The routing problem becomes more significant in this scenario, where resources are scarce and the routing protocol must be able to react to frequent topological changes and traffic variability without introducing excessive control overhead. In this situation, guaranteeing End-to-End QoS from the perspective of the network level is a difficult task.

In a wireless environment, the number of users that try to access is generally higher than the available radio resources. On the other hand an appropriate

\footnotetext{
* This work was financed by the Spanish Government (Project TEC2004-04529/TCM from MEC and FEDER), Gobierno de Aragón for WALQA Technology Park and the European IST Project PULSERS Phase II (IST - 027142).
} 
resource reservation according to the demands is required to obtain the desired QoS. Under these conditions, solving the trade-off between guaranteeing the requirements for the QoS provision with the highest efficiency in the use of the network resources is essential to maximize the system capacity. QoS support in MANETs involves the whole protocol stack of the network infrastructure [1. One of the most promising ways to achieve the trade-off between the QoS provision and the efficient resource utilization is the cross-layer design (Fig. 1), which allows different layers to share network status information in order to cooperate in the network resource management.

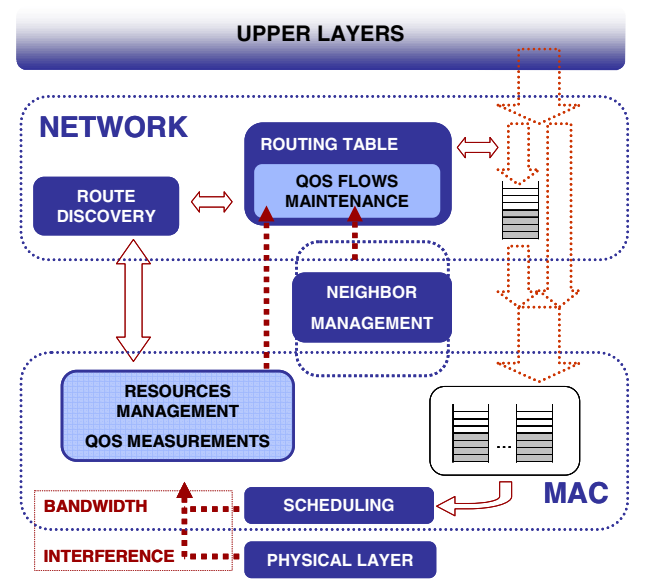

Fig. 1. Cross-layer Design for QoS Routing. General Architecture

The scope of most QoS Routing proposals only covers theoretical or heuristic approaches [23], without taking into account the potential problems of a practical implementation. Considering a realistic wireless ad hoc environment entails important physical implications that cannot be ignored to ensure the network capability to guarantee the demanded QoS. The interfering nature of the wireless medium and the lost of connectivity due to mobility affect any practical implementation, since collision-free transmissions cannot be assumed any more. This fact is especially critical on the control MAC level operations, such as connectivity maintenance or resource management. In order to validate any cross-layer proposal, it is necessary to consider the real implementation of the routing and $\mathrm{MAC}$ protocols to evaluate how they collaborate and the mechanisms that are actually considered to solve the QoS concept.

This work presents a QoS Routing based on a cross-layer operation that allows to identify the most appropriate path to cover the applications demands according to a new QoS metric. Thanks to the interaction between the routing and the MAC levels, this metric captures the resources availability, evaluating the capacity of providing QoS for an ongoing connection. The implemented architecture proposes the cooperation between a modified version of the Ad hoc 
On-demand Multipath Distance Vector Routing (AOMDV) 4 and the ADHOC MAC protocol [5]. This solution has been evaluated in a scenario where Signal to Interference Ratio (SIR) and mobility considerations have been taken into account to overcome the problems stemmed from this dynamic environment.

The remaining of the paper is organized as follows. Section 2 presents the basis of the ADHOC MAC protocol, and the proposed QoS Routing protocol is described in Section 3 detailing the resource management mechanism. The solutions to the problems stemmed from the interference nature of the medium are introduced in Section 4 and the proposals to adapt the QoS routing operation to a mobility scenario are explained in Section 5 . Simulations results are shown in Section 6. Finally, some conclusions are provided in Section 7 .

\section{The ADHOC MAC Protocol}

Determining the bandwidth availability in an ad hoc environment is not an easy task and it is basically dependent on the current MAC layer. In this proposal, a MAC TDMA layer based on the ADHOC MAC protocol has been considered. ADHOC MAC works on a slot synchronous physical layer and implements a completely distributed access technique capable of dynamically establishing a reliable single-hop Basic broadcast CHannel (BCH) for each active terminal. Each $\mathrm{BCH}$ carries signaling information (FI - Frame Information), including priorities, which provides a prompt and reliable distribution of layer-two connectivity information to all the terminals.

When dealing with multimedia applications, in response to the demanded QoS, the MAC level must efficiently allocate resources for several differentiated services. To this purpose, the access and reservation strategies proposed in 6 have been considered in order to provide a reservation based mechanism to handle the access to data user resources and a simple but efficient traffic differentiation by exploiting the in band signaling provided by the ADHOC MAC protocol. The basis of this strategy relies on the use of the $\mathrm{BCH}$ capabilities to signal the request before the access, in such a way that collisions can be theoretically avoided (Book In Advance Strategy - BIAS). Preemption can be carried out in order to allocate resources for high priority services despite the lower priority ones. The policy used to resolve the conflicts in reservation is explained in detail in 6 .

The ADHOC MAC protocol provides an efficient mechanism to measure the available bandwidth in terms of slots as well as ensures the reservation of the demanded ones. The core of the operation is the status information maintained by each terminal for all the slots in the TDMA frame. According to the information received in the $\mathrm{BCH}$ of each neighbor, related to the status they perceive (FREE, BUSY) a terminal defines its own slot status as RESERVED or AVAILABLE in order to select the resources to be allocated. This information is conditioned by the priority since higher priority services can steal resources to the lower ones. The complete rules to define this status are described in [6]. 


\section{The QoS Routing Algorithm}

A new QoS application is considered as a flow that needs a stable route during the whole connection. In the basic AODV (Ad hoc On-Demand Distance Vector [7] operation the source broadcasts requests packets $(R R E Q)$ referring this flow and each intermediate node rebroadcasts the first received copy of the RREQ until it reaches the destination, which sends a reply message (RREP) along the reverse path to the source. In terms of quality of service, several paths can satisfy the QoS requirements and the first request packet that reaches the destination does not actually identify the best path. The trade-off among different QoS requirements makes it difficult to choose the best solution. However, we can try to find a suboptimum path in terms of access delay but better satisfying the QoS requirements. The proposed QoS routing algorithm takes advantage of the multipath routing provided by the AOMDV to find several paths, although only one is selected according to a QoS metric based on the bandwidth requirements.

\subsection{QoS Metric: Path Bandwidth Calculation Process}

The AOMDV routing protocol has been adapted to include a modified version of the path bandwidth calculation algorithm described in 3 to measure the available bandwidth considering the whole path. The basic idea of this algorithm is to find the available TDMA slots that can be used for transmitting in every link along the path so that these slots, if reserved, would be interference-free. The measurement is performed and updated in each node during the discovery phase. The path bandwidth calculation ends in the destination node, and the calculated value represents the maximum available bandwidth between the source and the destination. The actual implementation of the algorithm operates as explained next.

According to the MAC level information, a node $k$ is aware of the available slots for transmitting without interfering other connections ( $S R T_{k}$ set) and the available ones for receiving without collision $\left(S R R_{k}\right.$ set). Since in the ADHOC MAC protocol, preemption of reserved slots with lower priority is possible when resources of high priority are demanded, the set of available slots for the new QoS flow in the routing level will include these lower priority slots as available. During $R R E Q s$ propagation, the set of available slots for communication in link $(i, j)$ is calculated in node $j$ and denoted as $P B_{i j}$. The set of transmitting slots must be disjoint in three consecutive hops to avoid collisions. According to this rule, each intermediate node appends its own $S R T$ to the $R R E Q$ packet, but also the $P B_{i j}$ calculated in the previous two hops. With this information, in addition to the $S R R$, the next node receiving this $R R E Q$ can calculate again the sets of slots to make them disjoint to the new link and update the appended information before forwarding the $R R E Q$. The number of available slots in each set is reduced to the minimum value in the three hops used to compute them. When the destination node receives the $R R E Q$, the dimension of the last availability set determines the total available bandwidth in the path. After waiting for several $R R E Q s$ (multipath), one of the paths that match the requirements is selected according 
to the metric. Then, the destination node sends the $R R E P$ packet through the reverse path to the source. The most updated information of the actual available set in every link is in the 3-hops-downstream neighbor. Therefore, to have an updated version of the available slots, during the reply phase a node appends to the RREP the more updated ones it has stored. When a node receives a $R R E P$, it updates the sets to be forwarded, but also selects the effective slots to transmit, according to the demands, from the available set in the corresponding link. Then, the BIAS mechanism of the ADHOC MAC protocol is performed to effectively reserve these selected slots. The proposed solution acts as a distributed Call Admission Control (CAC) performed during the discovery process of the routing protocol 8 . An example of the whole process is shown in Fig. 2

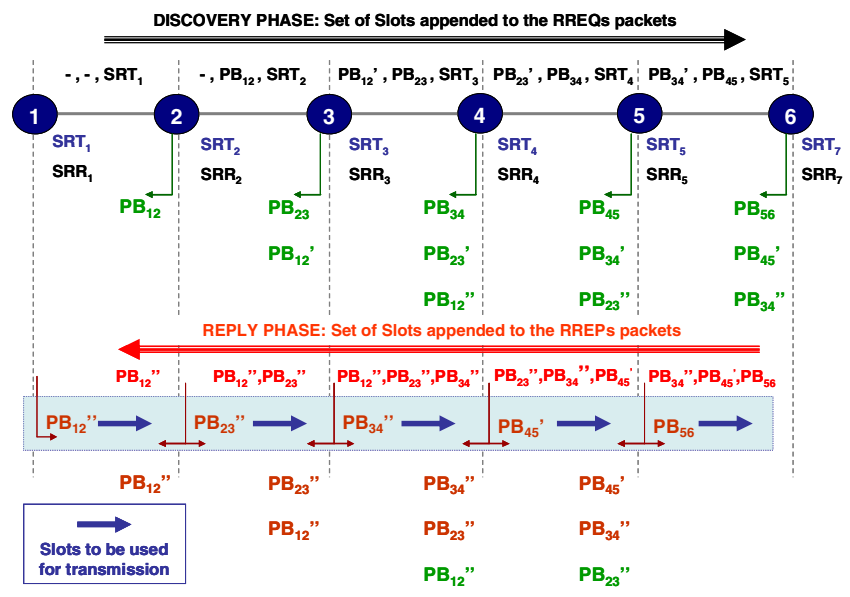

Fig. 2. Example of Path Bandwidth Calculation Process

The QoS metric computed along the path is given by (1)-(2):

$$
\begin{gathered}
B W_{\text {metric }}^{i, j}=\left|P B_{i, j}\right| \\
B W_{\text {metric }}^{P A T H}=\min \left(B W_{\text {metric }}^{i, j}\right)
\end{gathered}
$$

where $B W_{\text {metric }}^{i, j}$ is the measured $B W$ value in the previous link $(i, j)$, equal to the number of available slots (dimension of $P B_{i, j}$ ), and $B W_{\text {metric }}^{P A T H}$ is the more restrictive value in the whole path, equal to the last link $B W_{\text {metric }}$.

When an intermediate node receives a $R R E Q$ for a new flow, it updates the appended QoS metric in the $R R E Q$, and evaluates if the QoS requirements are met. Only those packets received from paths with a valid metric are forwarded. A $R R E Q$ message is dropped if $B W_{\text {metric }}^{i, j}<N_{R R E Q}$, the required slots. Repeated $R R E Q s$ are not directly dropped in the destination node in order to perform a multipath operation so that several paths can be discovered and finally one can be selected, corresponding to the highest $B W_{\text {metric }}^{P A T H}$ and the lowest number of hops. 


\section{Operation in an Interference-Aware Scenario}

In a typical scenario, connectivity among nodes is only based on Euclidean distances. In this situation, all nodes in the transmission range can correctly decode only one transmitted packet. If more than one neighbor transmits, a collision occurs. Transmissions one hop away are not sensed, which can lead to hidden-terminal [9] and exposed-terminal [10] problems, but if the MAC signaling can avoid them, as in the BIAS access scheme of the ADHOC MAC, totally collision-free transmissions are possible and the reuse capability is theoretically maximized.

However, in a more realistic scenario, where the actual interference produced by all the transmitting terminals is taken into account, collisions can still occur, leading to the loss of already reserved resources. The ability of decoding the received information is not only related to the distance of the transmitter and, in fact, the coverage range defined by one-hop neighbors varies according to the load conditions, as it is shown in Fig. 3. In this scenario, a transmission is considered successful if (3) is satisfied.

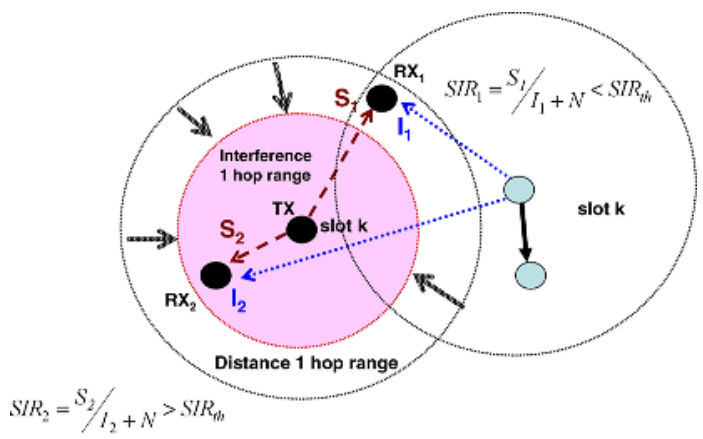

Fig. 3. Variations in the coverage range in a realistic interference scenario

$$
\begin{gathered}
S I R_{r x, i, j}^{k}=\frac{P_{t x, i}^{k} \cdot L_{i, j}}{P_{\text {int }}+P_{\text {noise }}}>S I R_{t h} \\
P_{\text {int }}=\sum_{n \in N_{t x}^{k}, n \neq i} P_{t x, n}^{k} \cdot L_{n, j}
\end{gathered}
$$

where $S I R_{r x, i, j}^{k}$ is the SIR received by terminal $j$ from terminal $i$ in slot $k, P_{t x, n}^{k}$ is the power transmitted by user $n$ in slot $k, L_{n, j}$ is the path loss between users $n$ and $j, P_{\text {int }}$ is the total interference produced by other transmitting terminals, $N_{t x}^{k}$ is the set of transmitting terminals in slot $k, P_{n o i s e}$ is the thermal noise and $S I R_{t h}$ is the minimum required SIR to be able to decode the information.

In order to provide more reliable link connections, the implemented MAC level includes a slot status, DIRTY, to identify slots where some power can be 
sensed, although the terminal is not capable of decoding any information. A terminal cannot transmit in a slot that the potential receiver signals as DIRTY, assuming that the reservation may fail due to interference. Anyway, despite the DIRTY slot, collisions can still occur, even in a signaled as FREE slot. The interference power can be sensed if it exceeds a certain threshold, the Carrier Sense Threshold $\left(C S_{t h}\right)$. However, given a $P_{i n t}<C S_{t h}$, which will lead to signal a slot as FREE, it can still happen that $S I R_{r x}<S I R_{t h}$, especially when two terminals are neighbors but with a little margin for additional interference. In addition, even when the slot is correctly reserved, any new activation, although established between a distant pair of nodes, increases $P_{i n t}$, which can disrupt the ongoing transmission.

The negative effect of the not sensed interference or that created by new distant connections is even more critical over the $\mathrm{BCH}$ transmissions, since the carried control information is the basis for performing an appropriate resources allocation (BIAS access scheme) and for maintaining updated connectivity information. In fact, the cooperation with the network level can lead to wrong routing decisions due to this degraded MAC operation. The variability in the network activity as a consequence of these nodes trying to reallocate a $\mathrm{BCH}$ makes it more difficult to efficiently perform the resource management.

In this scenario, links between nodes that are actually in the coverage limit, have a very low margin to overcome this potential unexpected interference, therefore they are more likely to fail. In order to decrease this failure probability, then reducing the instability of the network, these links should be avoided.

As a first approximation, a partial solution can be performed in the network layer, via a new QoS metric to be included in the routing process, in addition to the measurement of the bandwidth demands. This metric evaluates the capability of the links to overcome additional interference providing and additional margin over the minimum required SIR to make the decoding correctly. The interlayer operation allows to use physical information to identify the quality of the link according to the received power and the measured SIR.

In 11 we show that this proposal allows to improve the global performance, specially the obtained throughput and delay for the admitted connections. However, this solution is applied for the QoS flows, although, as commented before, the more crucial effect is the instability generated in the network due to frequent $\mathrm{BCH}$ failures. Then, not only the QoS DATA packets need to be transmitted over reliable links, but also, and even more, the particular MAC BCH transmissions. In fact, the weakness of a link can be considered as a local characteristic that can be measured in the physical level. In order to provide this reliability to all connections (and also for best-effort traffic), an alternative to the $S I R_{\text {metric }}$ is to transfer the same concept to the MAC level trying to provide a security margin to overcome the interference for any connection. An additional threshold $P_{r x, \min }$ is used to verify the reliability of a link. This value is considered as the minimum received power to provide a theoretical $S I R_{\min }$ some dBs higher than $S I R_{t h}$ in the absence of any interference. Therefore, when a link is established under this condition, the effective $S I R_{r x, i, j}^{k}$ can be lower than the $S I R_{m i n}$ due 
to the interference but still higher than the $S I R_{t h}$. In addition to (3), a reliable link must satisfy:

$$
P_{r x, i, j}^{k}=P_{t x, i}^{k} \cdot L_{i, j}>P_{r x, \min }
$$

Weaker links, considered as those with a theoretical $S I R_{r x}$ near $S I R_{t h}$, are avoided since they will probably fail with slight increments of the interference in their environment, whereas those links covering both conditions can support higher levels of interference.

\section{Mobility Management: QoS Monitoring}

Once a path is selected, the variability in the network conditions would make unfeasible to maintain this path without a mechanism of QoS monitoring and path updating. In the normal operation of the AODV routing protocol nodes react to broken links sending error messages to inform the neighborhood about this event. New discoveries arise, as soon as the involved nodes realize the phenomenon, but this mechanism only alerts about broken links, assuming the path is unviable, whereas in a QoS environment links can be still viable although the bandwidth is not enough for covering the demands of a specific connection. Therefore, the routing algorithm must be capable of differentiating both effects making the terminals react appropriately according to the specific event.

The proposed updating process is performed using certain routing information piggy-backed in the $D A T A-A C K$ packets, similar to that sent during the $R R E Q-R R E P$ phase, which allows to realize if the QoS constrains are not met anymore. Without calculating again a QoS metric, the sets of available slots are forwarded as in the discovery phase. These sets include the already reserved slots for this QoS flow. As a result, the process identifies the demanded bandwidth and the additional available one. Only when the interference has disrupted the reserved slots the piggy-backed $A C K$ acts as a $R R E P$ packet that invokes in the MAC level the reservation of the new slots. Therefore, new resources, if available, can be reallocated without discovering a new path. If the QoS cannot be maintained after several updating phases (the number is configurable), a $Q L O S T$ ( $Q O S L O S T$ ) packet is sent to the source in order to trigger the discovery of a new path capable of satisfying the demanded bandwidth. When dealing with a QoS lost, the proposed scheme tries to find a new available path with the demanded QoS as during the discovery of a new path. However, despite the degradation of the QoS, the former path is sill viable to send traffic as best-effort. Therefore, this path is maintained to avoid dropping packets in excess while discovering an alternative QoS path. The changes in topology can make unfeasible to reallocate the connection with the demanded QoS. Then, maintaining the previous path as active avoids blocking the connection once it has been admitted. This blocking is considered more harmful than not admitting a new flow, since an established connection would suffer a non-tolerable degradation. Unless a new path covering the requirements is found releasing the previous one, the best-effort route keeps the connection active. When the updating process is triggered again, successive attempts to reallocate resources can finally lead to provide the demanded QoS. 
When a broken link affects any path, a new discovery arises, as if it were a new connection to be admitted. However, if the affected path requires certain QoS, a new attempt to allocate resources may fail due to the new topology. Unlike the situation of the QoS lost, there is not a viable best-effort path to keep on sending packets. Then, if a new QoS path were not discovered, the connection would be blocked. To avoid this harmful degradation, the proposed QoS routing relaxes the constraints during the discovery of an already admitted but disrupted connection. The dropping policy applied by the intermediate nodes when receiving routing messages is not followed, in order to find the best available path, but including the best-effort alternative. The QoS metric is only used to classify the alternative paths. Anyway, if the connection is finally readmitted, the QoS updating process allows to subsequently reallocate resources if they are available. However, when the allocation of the disrupted connection is not possible, it is dropped to avoid colliding with the correctly dispatched ones.

\section{Performance Evaluation}

In order to evaluate the performance of the proposal, we have built up a simulator in $\mathrm{C}++$ which implements the functionalities of the design, considering the ADHOC MAC protocol interacting with the modified AOMDV, including the path-bandwidth calculation algorithm integrated in the routing process in a realistic ad-hoc environment. The connectivity among terminals is determined by the ability of decoding the $\mathrm{BCH}$ transmissions according to the received SIR, considering a transmitted power of $20 \mathrm{dBm}$, a Kammerman propagation model (6) and a minimum decoding threshold $S I R_{t h}$ of $5 \mathrm{~dB}$. The $S I R_{\text {min }}$ has been selected to $8.5 \mathrm{~dB}$ to overcome the problems of unexpected interference.

$$
L_{i, j}= \begin{cases}20 \cdot \log _{10}\left(\frac{4 \cdot \pi \cdot d}{\lambda}\right) & \text { if } d<8, \\ 58.3+33 \cdot \log _{10}\left(\frac{\mathrm{d}}{8}\right) & \text { if } d \geq 8\end{cases}
$$

A set of 50 nodes are randomly positioned within a square area of $2 \mathrm{Km}^{2}$. Terminals follow the Random Waypoint (RWP) mobility model [12. Several scenarios with average node speed of 1 and $9 \mathrm{~km} / \mathrm{h}$ are simulated and compared to the performance in static conditions. Pause time in the RWP model is fixed to 2 seconds. Connections between different pairs of nodes are generated according to a Poisson process with rate [connections/sec.] ranging according to the simulated offered load and the mean connection duration (210 sec.). QoS flows are generated as CBR sources demanding a bandwidth of $64 \mathrm{kbps}$ (2 TDMA slots). Packets exceeding a delay of $500 \mathrm{~ms}$. are early discarded. In order to evaluate the proposed QoS routing, the measured parameters are delay of correctly received packets and throughput, calculated as the ratio among dispatched and offered traffic expressed in packets.

Figures 4 and 5 show the performance of the proposed architecture. The cross-layer operation acts as a distributed CAC that allows to efficiently allocate resources for the different connections leading to a better distribution of the resources occupation in the network. 


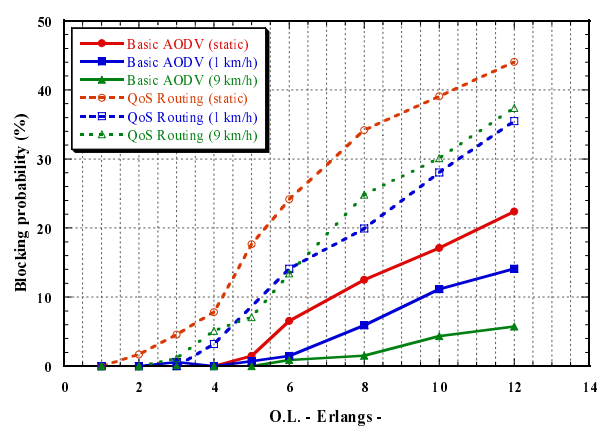

(a) Blocking probability

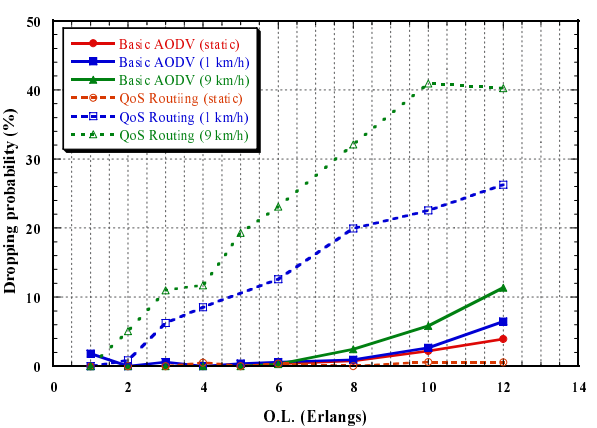

(b) Dropping probability

Fig. 4. Performance of the proposed QoS Routing vs. the basic AODV operation. Failure probability: blocking of new connections and dropping of disrupted admitted connections.

When a new application cannot be allocated, the proposed routing does not find any available path not admitting the connection, which increases the number of blocked connections compared to the basic operation, as it is shown in figure 4(a) Even, when changes in the topology makes the network distribution unable to efficiently allocate the admitted connections, the disrupted ones are discarded, leading to a failure probability (figure 4(b). However, this implies in fact a reduction on the congestion of the network which allows to better deal with the admitted connections, which experience lower delay, figure 5(a) and higher individual throughput, figure $5(\mathrm{~b})$.

The individual improvement, in the end, leads to a higher global performance, as observed in figure 6] which shows that, even in a high mobility scenario, the QoS Routing outperformes the basic AODV operation in terms of correctly

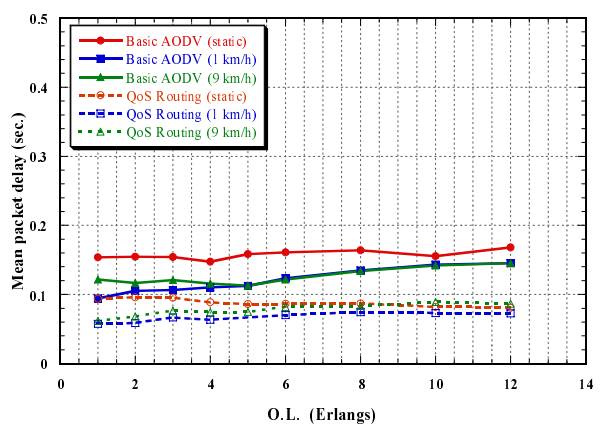

(a) Delay of correctly received packets

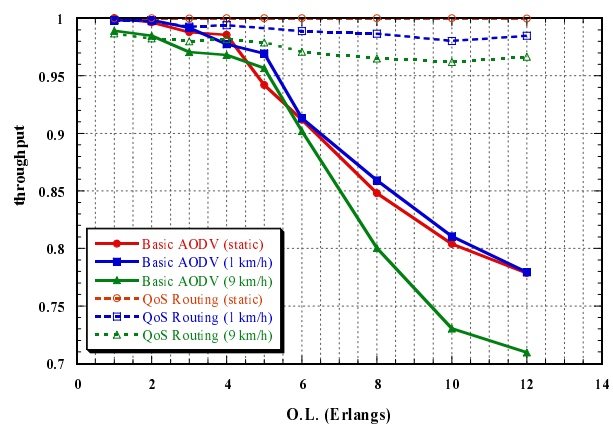

(b) Throughput of correctly admitted connections

Fig. 5. Performance of the proposed QoS Routing vs. the basic AODV operation. Evaluation of obtained the QoS for admitted connections (mean throughput and delay). 


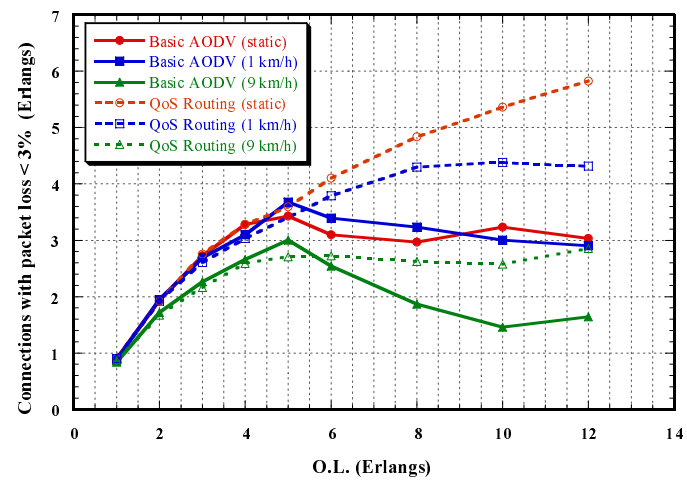

Fig. 6. Correctly dispatched connections with packet loss $<3 \%$

dispatched connections. A connection is considered correctly dispatched if it is not dropped and it achieves its expected QoS. The packet delay is limited thanks to the discarding policy, but the packet loss must be limited to the demanded restriction (considered $3 \%$ in the simulations).

The better resource allocation is responsible of the packet discarding reduction, but its effectiveness in a mobility scenario is achieved thanks to the implemented QoS updating process. When a connection is admitted, the initial resource allocation allows to ensure certain bandwidth avoiding discarding packets due to congestion. However, in the dynamic environment, resources need to be reallocated due to the QoS lost. Thanks to the temporary maintenance of besteffort paths upon reception of a $Q L O S T$ message, the packets dropping probability is reduced. While trying to rediscover a route with enough bandwidth, packets are still sent using these best-effort paths. Only when the allocation of new resources is not possible in the new topology, the connection is dropped in order to avoid disrupting the other ones. In the end, the response of the protocol to the interference variability (mobility / load increase) allows to maintain the desired QoS for the correctly dispatched connections.

\section{Conclusions}

This paper presents a QoS Routing based on a cross-layer architecture acting as a distributed admission control capable of efficiently allocating resources for bandwidth demanding applications. This proposal has been designed to overcome the problems stemmed from the interference nature of the wireless medium and the dynamic environment of mobile ad hoc networks.

The obtaining throughput gain of the proposed scheme is reduced as the nodes speed increases but in any case, this solution outperforms the basic AODV operation for any traffic load condition. The evaluation of the proposal in a realistic interference and mobility scenario has shown the capability of the cross-layer operation to react to the environment providing a soft-QoS even in a dynamic situation. 


\section{References}

1. Mohapatra, P., Li, J., Gui, C.: QoS in mobile ad hoc networks. IEEE Wireless Communications (June 2003) 44-52

2. IEEE Journal on Selected Areas in Communications.: Special Issue on Wireless Ad Hoc Networks. Volume 17. (August 1999)

3. Zhu, C., Corson, M.: QoS routing for mobile ad hoc networks. In: Proceedings of the 21st Annual Joint Conference of the IEEE Computer and Communications Societies (INFOCOM'02). Volume 2., New York, USA (June 2002) 958-967

4. Marina, M., Das, S.: On-demand multi path distance vector routing in ad hoc networks. In: Proceedings of the International Conference on Network Protocols ICNP'01, Riverside, California, USA (2001) 14-23

5. Borgonovo, F., Capone, A., Cesana, M., Fratta, L.: ADHOC MAC: a new MAC architecture for ad hoc networks providing efficient and reliable point-to-point and broadcast services. Wireless Networks (WINET) 10(4) (July 2004) 359-366

6. Gállego, J.R., Canales, M., Hernández, A., Campelli, L., Cesana, M., Valdovinos, A.: Performance evaluation of point-to-point scheduling strategies for the ADHOC MAC protocol. In: Proc. 8th International Symposium on Wireless Personal Multimedia Communications (WPMC'05), Aalborg (Denmark) (2005) 1380-1384

7. Perkins, C.E., Belding-Royer, E.M., Das, S.: Ad Hoc On-Demand Distance Vector (AODV) Routing. Experimental RFC 3561 (July 2003)

8. Canales, M., Gállego, J.R., Hernández, A., Valdovinos, A.: Cross-layer proposal for QoS routing in mobile ad-hoc networks. In: Proc. 8th International Symposium on Wireless Personal Multimedia Communications (WPMC'05), Aalborg (Denmark) (2005) 1325-1329

9. Tobagi, F.A., Kleinrock, L.: Packet switching in radio channels. part 2. The hidden terminal problem in carrier sense multiple-access and the busy-tone solution. IEEE Transactions on Communications 23(12) (December 1975) 1417-1433

10. Haas, Z.J., Deng, J.: Dual Busy Tone Multiple Access (DBTMA): A multiple access control scheme for ad hoc networks. IEEE Transactions on Communications 50(6) (June 2002) 975-985

11. Canales, M., Gállego, J.R., Hernández, A., Valdovinos, A.: Interference-aware routing with bandwidth requirements in mobile ad hoc networks. In: Proc. IEEE 62nd Semiannual Vehicular Technology Conference (VTC2005-fall), Dallas (USA) (2005)

12. Johnson, D.B., Maltz, D.A.: Chapter 5. In: Dynamic source routing in ad hoc wireless networks. Kluwer Academic Publishers (1996) 153-181 